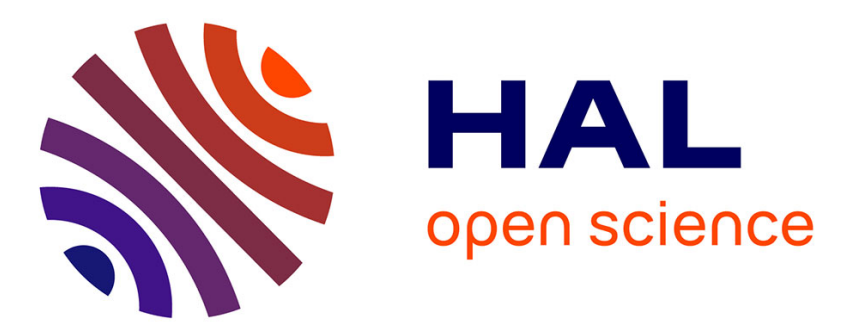

\title{
Hand-eye self-calibration of an ultrasound image-based robotic system
}

Marie-Aude Vitrani, Guillaume Morel

\section{To cite this version:}

Marie-Aude Vitrani, Guillaume Morel. Hand-eye self-calibration of an ultrasound image-based robotic system. 2008, 10.1109/IROS.2008.4651013 . hal-01170721

\section{HAL Id: hal-01170721 \\ https://hal.sorbonne-universite.fr/hal-01170721}

Submitted on 2 Jul 2015

HAL is a multi-disciplinary open access archive for the deposit and dissemination of scientific research documents, whether they are published or not. The documents may come from teaching and research institutions in France or abroad, or from public or private research centers.
L'archive ouverte pluridisciplinaire HAL, est destinée au dépôt et à la diffusion de documents scientifiques de niveau recherche, publiés ou non, émanant des établissements d'enseignement et de recherche français ou étrangers, des laboratoires publics ou privés. 


\title{
Hand-Eye Self-Calibration of an Ultrasound Image-Based Robotic System
}

\author{
Marie-Aude Vitrani and Guillaume MorelUniversite Pierre et Marie Curie - Paris 6 - Paris Universitas \\ ISIR - Institut des Systmes Intelligents et de Robotique \\ 4 place jussieu, \\ 75005 PARIS, France \\ \{vitrani, morel\}@robot.jussieu.fr
}

\begin{abstract}
In recent years, there has been an increasing interest in developing systems that couple a robotic device with an ultrasound imager. Applications range from automatic probe positioning to ultrasound image-based guidance of instruments. One issue in these systems is to determine, prior to the intervention, the localization of the probe with respect to the robot. Literature suggests using external localizers, but they add to the complexity of the system, and the resulting precision is usually not good due to the addition of errors in the kinematic chain.

In this paper, we study the hand-eye calibration problem without using any additional localizers. A generic system consisting of a fixed probe observing an instrument manipulated by a robot is used. We first derive a simplified model for mapping the image of the instrument with its 3D location w.r.t. the probe and then propose a calibration procedure based on a minimization algorithm.

Results show that although very simple models were used for the imaging device, the localization is quite precise, as it results in errors of less than $2 \mathrm{~mm}$, which is enough of a number of ultrasound guided interventions.
\end{abstract}

\section{INTRODUCTION}

Because it is portable, real time, inexpensive and not irradiating, ultrasonic imaging is by far the most widely used medical imaging modality. Ultrasound imaging systems are used either for non invasive observation only, or for monitoring an intervention. In the first case, the practitioner has to precisely and stably positions the probe so as to obtain the desired cross-section for proper observation. In the latter, the surgeon moves an instrument inside a patient while observing the motion of the instrument with an outer ultrasound probe. In general, surgeon positions the probe in order to see the instrument and the target in the same image. Then he uses one hand to maintain the probe fixed in that position while with the other hand he manipulates the instrument toward the target. This requires high skills for coordinating the hands holding the instrument and the probe. In fact, the ultrasound image gives only a 2D cross-section of the 3D operating region, containing no depth information. Therefore, manual ultrasound guided interventions are limited to simple tasks, such as puncture using a needle [1].

Within this context, robotic systems are being developed in order to accomplish ultrasound image-based guidance in a more performing way than manual procedures. More and more interventional systems exploit both robotics and medical imaging.

A major issue in these systems is the registration of the different devices that is necessary to perform precise positioning.

A first solution consists of mechanically attaching the instrument holder with the probe. For example, a system for guiding a needle by visual servoing is proposed in [2]. It consists of an ultrasound probe and a two degrees of freedom needle manipulator. The needle manipulator is mechanically forced to remain in the probe plane. The needle orientation and penetration are directly known from the manipulator joint position, thus not requiring any external registration. However, this approach limits the applications to those where the range of motion of the instrument with respect of the probe is small. It is thus not appropriate for applications where the probe location and the instrument port are to be chosen independently by the surgeon. On the contrary, in this research, we target applications where:

1) the relative localization of the probe holder and the instrument holder are to be setup independently;

2) the instrument has more than one or two degrees of freedom w.r.t. the probe.

A typical application described in [3], [4] consists of intracardiac surgery, where an instrument, manipulated by a robot, is inserted into the beating heart, where it can move with four degrees of freedom while its motion is observed through a trans-esophagus ultrasonic probe. By no way the probe can be physically linked to the instrument.

To cope with this type of registration need, there has been a significant effort towards 3D reconstruction, associated with 3D imaging. In this class of systems, pre-or peroperative images are processed in order to reconstruct 3D information which is then used by the robot. In [5], two robots collaborate. A first robot handles the ultrasound probe. Thanks to a localization of the anatomical target in the image and geometrical model, a second robot, handling a needle, is commanded towards the computed 3D position. In vitro tests emphasize a better accuracy than with manual procedures. The system proposed by [6] allows carrying out a biopsy of the prostate. The prostate 3D model is reconstructed using the ultrasound images. Thus the robot is fed with the 3D model in order to compute the trajectory. The tests on dead 
bodies showed a final positioning error of about, at best, $2.5 \mathrm{~mm}$. Some systems also use the images to perform, in real time, the 3D reconstruction of the desired position instead of establishing a patient model from preoperative images, [7], [8]. In these systems, the target is detected and tracked in the images. Their 3D position can then be calculated in a robot coordinate frame. The main limits of these approaches are:

1) external localizers are used for the registration of the devices, which adds to the complexity and price of the overall system, while it is desirable to limit the amount of equipment brought into the operating room;

2) the final geometrical positioning algorithm consists of chaining several transforms, from the probe to the robot base, then from the robot base to the instrument, which results in significant errors.

Avoiding the accumulation of geometrical errors is always possible by closing the loop, namely positioning the instrument using visual servoing. In this case, corrections are constantly made so that the instrument location in the image is finally correct, whatever the geometrical errors are, thanks to the capacity of rejecting static disturbances. However, apart from the specific visual servoing technique that we proposed in [4], all the other existing techniques (see e.g. [9], [10]) require the knowledge of the location of the probe with respect to the instrument.

Therefore, there is a common need for a registration procedure that avoids the use of external sensors. In [11], such a calibration is proposed by the use of a phantom visible in the ultrasound $2 \mathrm{D}$ cross section, resulting in errors as small as $2.5 \mathrm{~mm}$ for a focus depth of $6 \mathrm{~cm}$. This approach is however not valid for the type of targeted applications, since the phantom could not be used in an operating room.

In this paper, we have used a conventional hand-eye calibration procedure, which, to our knowledge, had never been used so far with ultrasound imaging. Its principle is to identify the unknown parameters of a measurement equation, thanks to several image measures taken at several positions of the instrument w.r.t. the probe. Only the relative motion of the instrument is known, thanks to the instrument holder proprioceptive sensors. In the case of ultrasound imaging, the difficulty lies not only in the image noise, which is high, but also in the fact that the imaging geometry is rather complex, as the probe produces a cross section over a slice of unknown and depth-varying thickness. This hardly translates into parametric equations. We thus used a simplified model with a planar cross section, arbitrarily identifying the center of image blob to the image of a $3 \mathrm{D}$ point belonging to a known straight line of the instrument. In spite of this drastic simplification, after calibration, the remaining error between the real measurements and the model remains less than 2 pixels.

The rest of the paper is organized as follows: Section II describes the canonical system used to demonstrate the approach and its simplified model. In section III, the calibration algorithm is detailed. Finally, Section IV shows the experimental validation.

\section{ULTRASOUND BASED ROBOTIC SYSTEM}

\section{A. System overview}

A canonical configuration of a robot-held instrument guided through US imaging has been used for this study. It is sketched in Fig. 1. The robot-held instrument is observed by an ultrasound probe, which images are transmitted in real time to a computer.

The instrument is attached to the end-effector of the robot in such a way that its axis is parallel to the axis of the last robot joint denoted $\mathbf{k}_{\mathbf{6}}$ using Denavit-Hartenberg convention. Furthermore, the origin $\mathrm{O}_{6}$ of the frame attached to the robot end-effector belongs to the instrument axis.

We assume that the probe is fixed with respect with the robto base. Indeed, during US-guided intervention, the instrument is moved while the probe stays still. Moving at the same time instrument and probe requires too high skills of coordination and could lead to loose the visibility of the target or instrument.

In the chosen configuration, the instrument axis intersects the ultrasound plane. Thus, a blob is visible in the image, which allows for the instrument localization, thanks to appropriate image processing algorithms.

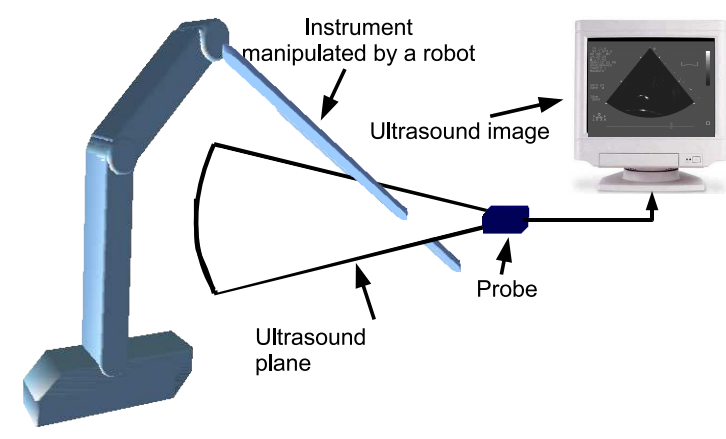

Fig. 1. System Description

This algorithm provides in real time the coordinates $\mathbf{s}_{m e s}$ of the center of gravity $G$ of the blob corresponding to the instrument image see [12].

\section{B. Geometrical modelling}

In order to derive the identification algorithm, a first step is to establish a geometrical model between the $3 \mathrm{D}$ position and orientation of the instrument and its 2D position in the image. Literature and documentation report that the points visible in the US image are those belonging to the intersection of the visualized object and a 3D thick beam, as illustrated in Fig. 2. Therefore, the relationship between the position and orientation of the instrument and the center of gravity of the image blob, grouping all the visible points after image binarization, is quite complex to derive. We thus propose a simplification consisting of identifying the center of gravity $G$ of the instrument image and the point $M$ corresponding at 
the intersection of a line $\mathscr{D}$ (which represents the axis of the instrument) and a plan $\Pi$ representing the ultrasound probe, see Fig. 2.

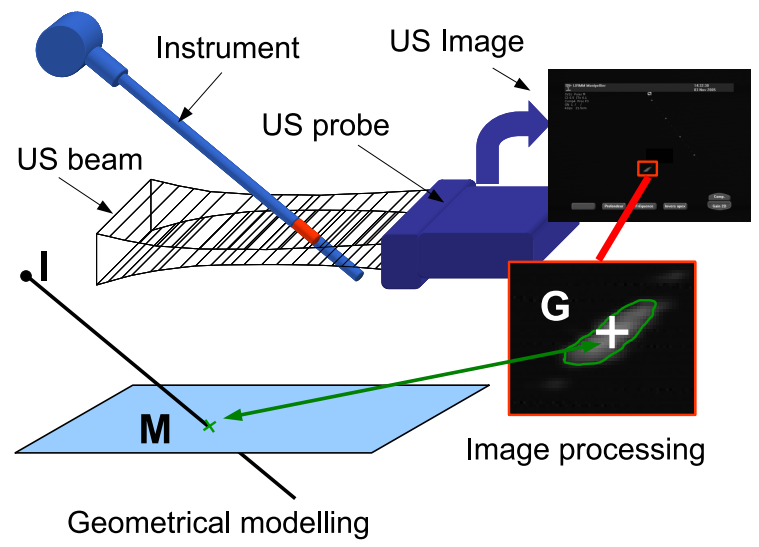

Fig. 2. Modelling hypothesis

The following coordinate frames are used for the geometrical modelling (see Fig. 3):

- $\mathscr{F}_{0}=\left\{O_{0} ; \mathbf{i}_{\mathbf{0}}, \mathbf{j}_{\mathbf{0}}, \mathbf{k}_{\mathbf{0}}\right\}$ is the coordinate frame attached to the robot base.

- $\mathscr{F}_{6}=\left\{O_{6} ; \mathbf{i}_{\mathbf{6}}, \mathbf{j}_{\mathbf{6}}, \mathbf{k}_{\mathbf{6}}\right\}$ is the coordinate frame attached to the robot end-effector.

- $\mathscr{F}_{I}=\left\{I ; \mathbf{i}_{\mathbf{I}}, \mathbf{j}_{\mathbf{I}}, \mathbf{k}_{\mathbf{I}}\right\}$ is the coordinate frame attached to the instrument $\mathscr{I}$, with $I$ belonging to the instrument axis and the vector $\mathbf{k}_{\mathbf{I}}$ being parallel to the instrument axis. The assembly described previously allows to choose $\mathbf{k}_{\mathbf{I}}=\mathbf{k}_{\mathbf{6}}$ and $I=O_{6}$.

- $\mathscr{F}_{P}=\left\{P ; \mathbf{i}_{\mathbf{P}}, \mathbf{j}_{\mathbf{P}}, \mathbf{k}_{\mathbf{P}}\right\}$ is the coordinate frame attached to the ultrasound probe $\mathscr{P}$ with $\mathbf{k}_{\mathbf{P}}$ perpendicular to the ultrasound plane, and $P$ the origin of the ultrasound beam.

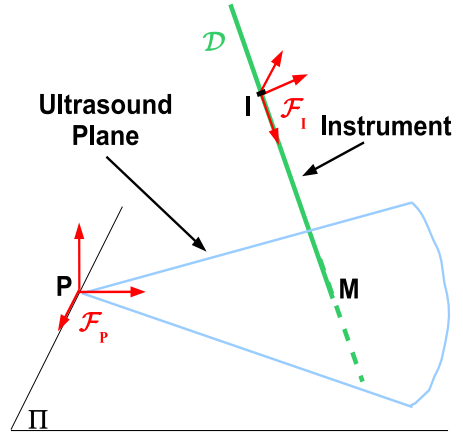

(a) Coordinate frames description

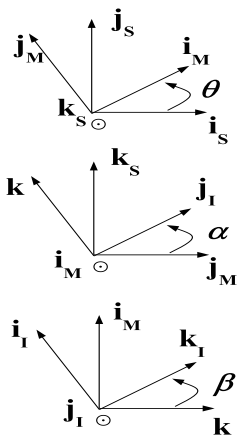

(b) Euler's angles
Fig. 3. Coordinate frames and angles used

Deriving the geometrical model consists in finding the coordinate of the point $M$ in the frame attached to the probe. The point $M$ belongs to the plane $\Pi$ as well as to the line $\mathscr{D}$.
Since point $M$ belongs to line $\mathscr{D}$, one has :

$$
\left\{\begin{array}{l}
\mathbf{d}_{\mathbf{P M}}{ }^{T} \mathbf{k}_{\mathbf{P}}=0 \\
\mathbf{d}_{\mathbf{I M}}=l \mathbf{k}_{\mathbf{I}}
\end{array}\right.
$$

where, as in the rest of the paper, $\mathbf{d}_{\mathbf{A B}}$ denotes the vector from point $A$ to point $B$. Projecting in the probe frame $\mathscr{F}_{P}$ one gets:

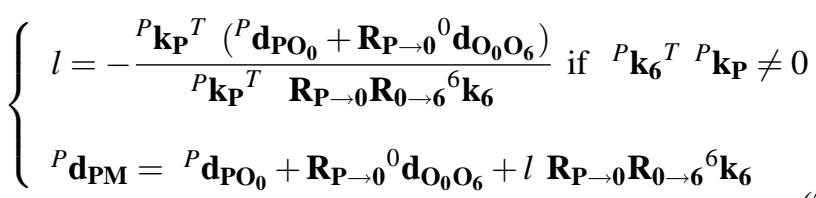

where, as in the rest of the paper, ${ }^{A} \mathbf{b}$ denotes the components of a vector $\mathbf{b}$ is the basis of frame $\mathscr{F}_{A}$ and $\mathbf{R}_{\mathbf{A} \rightarrow \mathbf{B}}$ is the rotation from frame $\mathscr{F}_{A}$ to frame $\mathscr{F}_{B}$. It can be noticed that the condition ${ }^{P} \mathbf{k}_{\mathbf{6}}{ }^{T}{ }^{P} \mathbf{k}_{\mathbf{P}} \neq 0$ means that the instrument and the plane $\Pi$ intersect in one point.

\section{Parameters to be identified}

The proposed geometric model depends on the geometrical model of the robot $\left({ }^{0} \mathbf{d}_{\mathbf{O}_{\mathbf{0}} \mathbf{O}_{\mathbf{6}}}\right.$ and $\left.\mathbf{R}_{\mathbf{0} \rightarrow \mathbf{6}}\right)$ and on the location of its base with respect to the probe $\left({ }^{S} \mathbf{d}_{\mathbf{S O}_{\mathbf{0}}}\right.$ and $\left.\mathbf{R}_{\mathbf{S} \rightarrow \mathbf{0}}\right)$.

Since a very precise industrial robot (Staubli TX40) is used in these experiments, one can assume that the position ${ }^{0} \mathbf{d}_{\mathbf{O}_{\mathbf{0}} \mathbf{O}_{\mathbf{6}}}$ and orientation $\mathbf{R}_{\mathbf{0} \rightarrow \mathbf{6}}$ of its end-effector with respect to its base are perfectly known. Moreover, we know that ${ }^{S} \mathbf{k}_{\mathbf{P}}={ }^{6}$ $\mathbf{k}_{\mathbf{6}}=\left[\begin{array}{lll}0 & 0 & 1\end{array}\right]^{T}$.

The unknown parameters of the model are thus the parameters describing the position and orientation of the base of the robot with respect to the probe: ${ }^{P} \mathbf{d}_{\mathbf{P O}_{0}}$ and $\mathbf{R}_{\mathbf{P} \rightarrow \mathbf{0}}$.

Finally, it should be noted that the measures in the image are extracted in pixels, therefore they must be converted into meters to fit in the geometrical model. The relationship between measures of pixels coordinates $\mathbf{s}_{\text {mes }}$ of the blob center of gravity and its coordinates in meters ${ }^{P} \mathbf{d}_{\mathbf{P M}}$ in the frame attached to the probe writes:

$$
\mathbf{s}_{\text {mes }}=\left[\begin{array}{ccc}
k_{x} & 0 & 0 \\
0 & k_{y} & 0
\end{array}\right]{ }^{P} \mathbf{d}_{\mathbf{P M}}
$$

with $k_{x}$ and $k_{y}$ being scale change gains to apply along $\mathbf{i}_{\mathbf{P}}$ and jp respectively.

The vector which contains all the uncertain parameters writes:

$$
\mathbf{p}=\left[\begin{array}{llllllll}
{ }^{P} x_{O_{0}} & { }^{P} y_{O_{0}} & { }^{P} z_{O_{0}} & r_{x} & r_{y} & r_{z} & k_{x} & k_{y}
\end{array}\right]^{T}
$$

with:

- the vector ${ }^{P} \mathbf{d}_{\mathbf{P O}_{\mathbf{0}}}=\left[\begin{array}{lll}{ }^{P} x_{O_{0}} & { }^{P} y_{O_{0}} & { }^{P} z_{O_{0}}\end{array}\right]^{T}$ being the position of the base frame origin expressed in the probe frame;

- the angles $r_{x}, r_{y}$ and $r_{z}$ being the XYZ Euler angles which describe the orientation of the base frame with respect to the probe frame. 


\section{CALIBRATION PROCEDURE}

\section{A. Principle}

The calibration procedure is to optimize a set of badlyknown parameters $\mathbf{p}$ of a system by minimizing the error between a set of measured variables $\mathbf{S}_{\text {meas }}$ with the same variables reconstructed $\mathbf{s}_{\mathbf{r e c}}$ using a model $\mathbf{s}=f(\mathbf{p})$. More precisely the principle of calibration is as follows:

- The system is placed in $n$ configurations. For each configuration $i \in\{1 . . n\}$, variables $\mathbf{S}_{\text {meas,i }}$ are measured;

- Badly-known parameters are optimized so as to minimize the error between the measured variables and the variables computed by the model;

- Once the best parameters obtained, the calibration is validated through a set measures that have not been used for optimization.

The way to optimize the parameters is described below: For the $\mathrm{i}^{\text {th }}$ location of the instrument, one computes the Jacobian matrix that maps the temporal derivatives of the parameters to be identified into the temporal derivatives of the measures. This relationship is written below:

$$
\dot{\mathbf{s}}_{\text {meas }, \mathrm{i}}=\mathbf{J}_{\mathbf{p i}} \dot{\mathbf{p}}
$$

It is a nonlinear problem of optimization since the matrix $\mathbf{J}_{\mathbf{p i}}$ depends on the parameters $\mathbf{p}$. However, an iterative procedure using a linearized relation provides good results, [13], providing that the initial guess is not too far from the real value.

Assuming small variation of the parameters $\delta \mathbf{p}$, one can write:

$$
\delta \mathbf{s}_{\text {meas,i }}=\mathbf{J}_{\mathbf{p i}} \delta \mathbf{p}
$$

For the $n$ measures, this equation is:

$$
\delta \mathbf{s}=\mathbf{J}_{\mathbf{p}} \delta \mathbf{p}
$$

with $\delta \mathbf{s}=\left[\begin{array}{llll}\delta \mathbf{s}_{\text {meas }, 1}{ }^{T} & \delta \mathbf{s}_{\text {meas }, 2}{ }^{T} & \ldots & \delta \mathbf{s}_{\text {meas, } n}{ }^{T}\end{array}\right]^{T}$ and $\mathbf{J}_{\mathbf{p}}=$ $\left[\begin{array}{llll}\mathbf{J}_{\mathbf{p} 1}{ }^{T} & \mathbf{J}_{\mathbf{p} \mathbf{2}}{ }^{T} & \ldots & \mathbf{J}_{\mathbf{p n}}{ }^{T}\end{array}\right]^{T}$.

This matrix equation is then solved in the least squares sense. Thus, if the matrix $\mathbf{J}_{\mathbf{p}}$ has a maximal rank, the inverted relation is:

$$
\delta \mathbf{p}=\mathbf{J}_{\mathbf{p}}^{+} \delta \mathbf{s}
$$

with $\mathbf{J}_{\mathbf{p}}^{+}=\left(\mathbf{J}_{\mathbf{p}}{ }^{T} \mathbf{J}_{\mathbf{p}}\right)^{-1} \mathbf{J}_{\mathbf{p}}{ }^{T}$ being the $\mathbf{J}_{\mathbf{p}}$ pseudo-inverse.

The following algorithm is then used to minimize the error between the variables $\mathbf{S}_{\mathbf{r e c}}$ reconstructed from the model and the variables $\mathbf{s}_{\text {mes }}$ measured:

$$
\begin{aligned}
& \mathbf{p}=\mathbf{p}_{\text {init }} \\
& \text { do } \\
& \quad \mathbf{s}_{\text {rec }}=f(\mathbf{p}) \\
& \quad \delta \mathbf{p}=\mathbf{J}_{\mathbf{p}}^{+}\left\|\mathbf{s}_{\text {rec }}-\mathbf{s}_{\text {meas }}\right\| \\
& \quad \mathbf{p}=\mathbf{p}+\delta \mathbf{p} \\
& \text { while }\left(\mathbf{s}_{\text {rec }}-\mathbf{s}_{\text {meas }}>\mathbf{e}_{\text {max }}\right) \text { and }\left(\|\delta \mathbf{p}\|>\delta \mathbf{p}_{\text {max }}\right)
\end{aligned}
$$

with $\mathbf{p}_{\text {init }}$ being an estimate value for the parameters to be optimized, $f(\mathbf{p})$ being the model, $\mathbf{e}_{\max }$ the error under which one the founded parameters are assumed to be the optimized ones and $\delta \mathbf{p}_{\max }$ the parameters variation under which one a local minimum is reached.

\section{B. Derivation of the jacobian matrix}

For the $\mathrm{i}^{\text {th }}$ configuration of the system, the parameter jacobian matrix $\mathbf{J}_{\mathbf{p i}}$ is then computed by derivating the geometrical model with respect to the uncertain parameters:

$$
\dot{\mathbf{s}}_{\text {mes }, \mathrm{i}}=\left[\begin{array}{ccc}
\dot{k}_{x} & 0 & 0 \\
0 & \dot{k}_{y} & 0
\end{array}\right]{ }^{P} \mathbf{d}_{\mathbf{P M}}+\left[\begin{array}{ccc}
k_{x} & 0 & 0 \\
0 & k_{y} & 0
\end{array}\right]{ }^{P} \dot{\mathbf{d}}_{\mathbf{P M}}
$$

We denote :

$$
\left\{\begin{array}{l}
\mathbf{J}_{\mathbf{k}} \dot{\mathbf{p}}=\left[\begin{array}{ccc}
\dot{k}_{x} & 0 & 0 \\
0 & \dot{k}_{y} & 0
\end{array}\right]{ }^{P} \mathbf{d}_{\mathbf{P M}} \\
\mathbf{J}_{\mathbf{m i}} \dot{\mathbf{p}}=\underbrace{\left[\begin{array}{ccc}
k_{x} & 0 & 0 \\
0 & k_{y} & 0
\end{array}\right]}_{=\mathbf{K}}{ }^{P} \dot{\mathbf{d}}_{\mathbf{P M}}=\mathbf{K}^{P} \dot{\mathbf{d}}_{\mathbf{P M}}
\end{array}\right.
$$

Thus the paramaters' jacobian matrix $\mathbf{J}_{\mathbf{p i}}$ writes for the $\mathrm{i}^{\text {th }}$ configuration:

$$
\mathbf{J}_{\mathbf{p i}}=\left[\mathbf{J}_{\mathbf{k}}+\mathbf{J}_{\mathbf{m i}}\right]
$$

To derive the matrix $\mathbf{J}_{\mathbf{k}}$ is trivial :

$$
\mathbf{J}_{\mathbf{k}}=\left[\begin{array}{cccccccc}
0 & 0 & 0 & 0 & 0 & 0 & P_{x_{M}} & 0 \\
0 & 0 & 0 & 0 & 0 & 0 & 0 & P_{y_{M}}
\end{array}\right]
$$

To compute $\mathbf{J}_{\mathbf{m i}}$, one needs to calculate the temporal derivative components ${ }^{P} \dot{\mathbf{d}}_{\mathbf{P M}}$ with respect to the parameters $\delta \mathbf{p}$ :

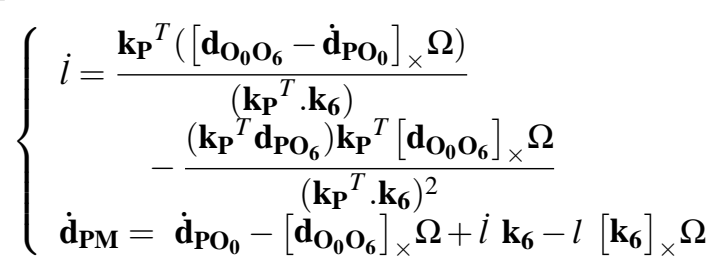

with $\Omega={ }^{P} \Omega(0 / P)=\left[\begin{array}{lll}\dot{r}_{x} & \dot{r}_{y} & \dot{r}_{z}\end{array}\right]^{T}$ and where all vectors are expressed in the coordinate frame $\mathscr{F}_{P}$.

One thus gets:

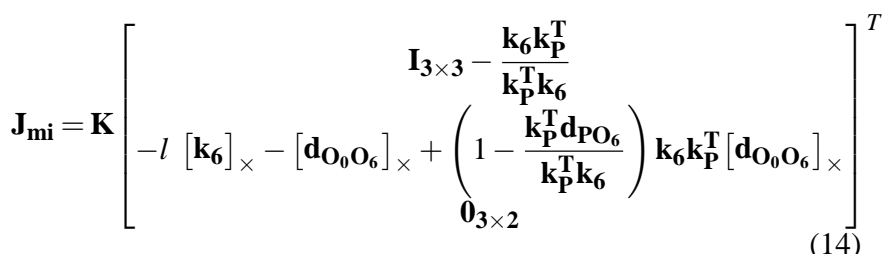

\section{EXPERIMENTAL VALIDATION}

\section{A. Experimental set-up}

The experimental set up, Fig. 4, is made of an ultrasound probe plunged into a tank of water. This probe provides images of an instrument manipulated by a 6 degrees of freedom robot, the Staubli TX40. The tip of the instrument is a $300 \mathrm{~mm}$ long thin cylinder with a $3 \mathrm{~mm}$ diameter. It consists of a steel rod covered with polyurethane. This guarantees a 
1) Experiment with a high intensity gain applied to the image: A second experiment is performed, applying higher gain intensity. The gain intensity modifies the form, the intensity and the size of the instrument track in the image as shown in Fig. 8. The previous experiment is performed again. The location of the probe with respect to the robot base is the same as previously, only the gain intensity is changed: $20 \mathrm{~dB}$ instead of $1 \mathrm{~dB}$.

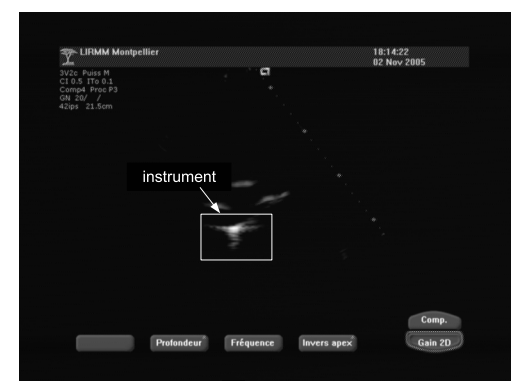

Fig. 8. Instrument image with $20 \mathrm{~dB}$ intensity gain

It is not necessary to optimize new parameters as they are not modified from the previous experiment. Only the comparison between measured and reconstructed position is needed, Fig. 9. The mean error is $0.68 \mathrm{~mm}$ in $\mathrm{x}$ and $0.83 \mathrm{~mm}$ in $\mathrm{y}$, with a maximal error of $1.68 \mathrm{~mm}$ in $\mathrm{x}$ and $1.65 \mathrm{~mm}$ in $\mathrm{y}$. These errors are equivalent to ones obtained during the first experiment (with a well settled ultrasound machine). The gain intensity does not modify the validity of the proposed model simplification. In other words, although the shape of the instrument image is modified, its center of gravity is unchanged. This is important because during in vivo experiments, high gains may be required to see anatomic features, [4].

2) Experiment with a short maximal depth visibility: A last experiment was conducted to illustrate that the maximum depth of visibility has no influence on the proposed model. In this aim, the previous experiment is carried out once more. The transformation between the robot base and the probe is
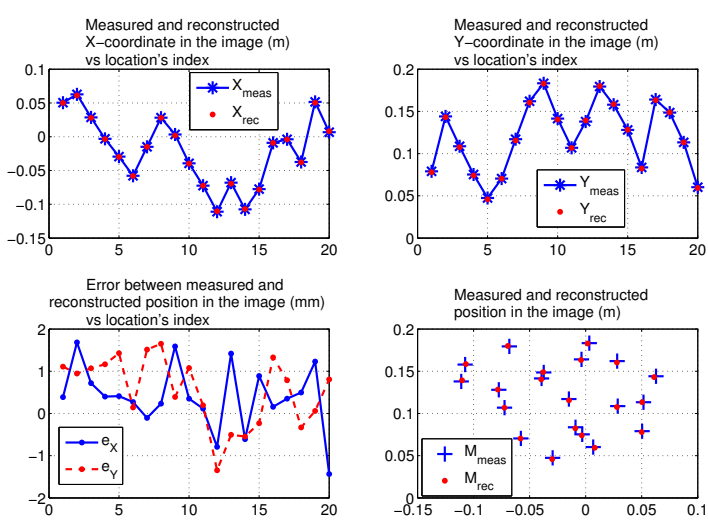

Fig. 9. Reconstruction results with $20 d B$ intensity gain unchanged, the intensity gain is $1 \mathrm{~dB}$, but the maximum depth of visibility is $10.8 \mathrm{~cm}$ instead of $21.5 \mathrm{~cm}$. This produces a zoom on the part of the image that is close to the origin of the ultrasound beam, Fig. 10.

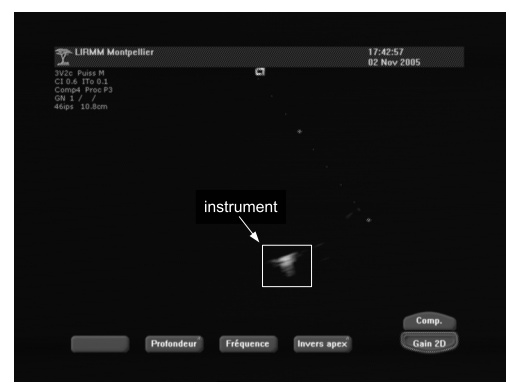

Fig. 10. Instrument image with short depth of visibility

As the visible area is smaller than in previous experience, only 9 positions were achieved. In addition, gains from scale change between meters and pixels are changed due to the new maximum depth of visibility. Therefore, a new identification of the parameters must be done.

After the reconstruction, results still emphasize a good precision as illustrated in Fig. 11. The mean error is $0.88 \mathrm{~mm}$ in $\mathrm{x}$ and $0.84 \mathrm{~mm}$ in $\mathrm{y}$, with a maximum error of $1.68 \mathrm{~mm}$ in $\mathrm{x}$ and $2.20 \mathrm{~mm}$ in y between the measured and rebuilt positions in the image. These errors are similar to those obtained with an ultrasound machine well settled. The maximum depth of visibility does not affect the validity of the proposed model. Furthermore, the optimized parameters are :

$$
\begin{gathered}
\left\{\begin{array} { l } 
{ S _ { x _ { O _ { 0 } } } = 2 9 . 7 6 \mathrm { cm } } \\
{ S _ { y _ { O _ { 0 } } } = 2 2 . 7 4 \mathrm { cm } } \\
{ S _ { z _ { O _ { 0 } } } = 2 5 . 0 2 \mathrm { cm } }
\end{array} \quad \left\{\begin{array}{l}
r_{x}=0.3788 \text { degrees } \\
r_{y}=-0.0215 \text { degrees } \\
r_{z}=175.3632 \text { degrees }
\end{array}\right.\right. \\
\left\{\begin{array}{l}
k_{x}=3.72 \mathrm{pixel} / \mathrm{mm} \\
k_{y}=3.57 \mathrm{pixel} / \mathrm{mm}
\end{array}\right.
\end{gathered}
$$

These results are consistent. Indeed, the geometrical configuration of the system has not been modified between the experiences, in particular, the position and orientation of the robot base with respect to the probe has remained constant. The optimization should provide the same orientation and position than previously which is the case as the error between the new optimized parameters and the previous ones is $\left[\begin{array}{lll}e_{x_{0}} & e_{y_{0}} & e_{z_{0}}\end{array}\right]=\left[\begin{array}{lll}-6 & -10 & 1\end{array}\right]$ millimeters and $\left[\begin{array}{lll}e_{r_{x}} & e_{r_{y}} & e_{r_{z}}\end{array}\right]=\left[\begin{array}{lll}-0.34 & 0.08 & -0.11\end{array}\right]$ degrees.

The errors on $e_{x_{0}}$ and $e_{y_{0}}$ are due to a shift of the origin of the image with respect to the ultrasound probe which in turn is due to the change in the depth gain.

Also, as the depth factor has been divided by 2 , it is not surprising that we identify that $k_{x}$ has been multiplied by 2.03 while $k_{y}$ has been multiplied by 1.97 , which gives a $1.5 \%$ error with respect to the gain change expectation.

Through these experiments, we validate the hypothesis which consists to match the center of gravity of a blob with the intersection between a line representing the instrument and a plane representing the ultrasound beam. Moreover, we demonstrate that the validity of this hypothesis does not 


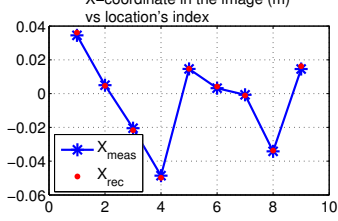

Error between measured and Visted position in the image $(\mathrm{mm})$

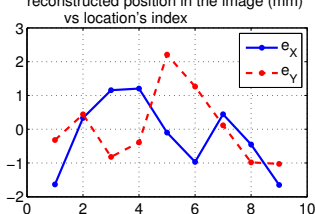

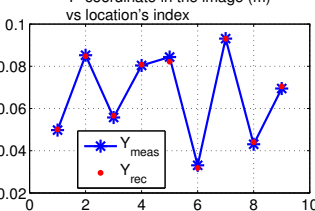

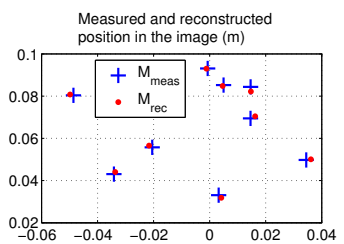

Fig. 11. Optimisation results with a short depth of visibility

depend on the tuning of the ultrasound machine.

\section{CONCLUSION}

Although we've been using a simple model together with a straightforward iterative optimization method, the results shown in this paper emphasize an unexpected precision, which, in certain cases, is as low as the US image resolution. Ultimately, since the precision is measured by matching a model to a set of measurements, a simple inverse model can be used to reach any desired position located in the image within a millimetric precision. Note that the presented setup was also used for real time control of the instrument using visual servoing, during in vivo beating heart experiments, [4].

\section{ACKNOWLEDGMENTS}

The financial support of the CNRS P.I.R. Robea program (project GABIE) is gratefully acknowledged. Thanks to the partners of this project: LIRMM, Montpellier, TIMC, Grenoble, CEA-FAR, CHUG, Grenoble, Pitié Salpêtrière, Paris.

\section{REFERENCES}

[1] L. Angelini and M. Caratozzolo. Intraoperative echography: the state of the art. Ann. Ital. Chir, 70(2):223-230, 1999.

[2] J. Hong, T. Dohi, M. Hashizume, K. Konishi, and N. Hata. An ultrasound-driven needle insertion robot for percutaneous cholecystostomy. Physics in Medicine and Biology, 49(3):441-455, 2004.

[3] M.-A. Vitrani, G. Morel, N. Bonnet, and M. Karouia. A robust ultrasound-based visual servoing approach for automatic guidance of a surgical instrument with in vivo experiments. In BIOROB'06, IEEE Int. Conf. on Biomedical Robotics and Biomechatronics, Pisa, Italy, February 2006.

[4] M.-A. Vitrani, H. Mitterhofer, G. Morel, and N.Bonnet. Robust ultrasound based visual servoing for beating heart intracardiac surgery. In Proc. of the ICRA 2007, Roma - Italy, April 2007.

[5] E.M. Boctor, G. Fischer, M.A. Choti, G. Fichtinger, and R.H. Taylor. A dual-armed robotic system for intraoperative ultrasound guided hepatic ablative therapy : A prospective study. In IEEE ICRA International Conference on Robotique and Automation, pages 2517-2522, New Orleans, LA, 2004.

[6] L. Phee, D. Xiao, J. Yuen, C. Fatt Chan, H. Ho, C. Hua Thng, C. Cheng, and W. Sing Ng. Ultrasound guided robotic system for transperinal biopsy of the prostate. In Proc. of the ICRA 2005, pages 1315-1320, 2005.

[7] J. Stoll, P. Dupont, and R. Howe. Ultrasound-based servoing of manipulators for telesurgery. In Telemanipulator and Telepresence Technologies VIII Conference, volume 4570 of Proceedings of SPIE, 2001.

[8] G. Megali, O. Tonet, C. Stefanini, M. Boccadoro, V. Papaspyropoulos, L. Angelini, and P. Dario. A computer-assisted robotic ultrasoundguided biopsy system for video-assisted surgery. In Medical Image Computing and Computer-Assisted Intervention - MICCAI 2001: 4th International Conference, Proceedings, pages 343-350, Utrecht, The Netherlands, 2001.

[9] A. Krupa, G. Fichtinger, and G.D. Hager. Full motion tracking in ultrasound using image speckle information and visual servoing. In Proc. of the ICRA 2007, Roma - Italy, April 2007.

[10] S.E. Salcudean, W.H. Zhu, P. Abolmaesumi, S. Bachmann, and P.D. Lawrence. A robot system for medical ultrasound. Robotics Research, May 2000.

[11] M.A. Janvier, F. Destrempes, G. Soulez, and G. Cloutier. Validation of a new 3d-us imaging robotic system to detect and quantify lower limb arterial stenoses. In 29th Annual International Conference of the IEEE Engineering in Medicine and Biology Society, EMBS 2007, pages 339-342, Lyon - France, 2007.

[12] T. Ortmaier, M.-A. Vitrani, G. Morel, and S. Pinault. Robust real-time instrument tracking in ultrasound images. In Proc. of SPIE Medical Imaging Conference, San Diego, California, USA, February 2005.

[13] W. Khalil and E. Dombre. Modlisation, identification et commande des robots. In Herms, $2^{\text {ime }}$ dition, 1999. 